\title{
FTMP-based Approach toward Dislocation Dynamics of PSB
}

\author{
S Ihara', N Kojima², T Hasebe ${ }^{3}$ \\ 1. Graduate School of Kobe University, Japan \\ 2. Koito Manufacturing CO., LTD., Japan \\ 3. Kobe University, Japan
}

\begin{abstract}
This study targets dynamic behavior of interacting dislocations associated with PSB (Persistent Slip Bands) ladder structures, ultimately responsible for fatigue crack initiations, based on FTMP (Field Theory of Multiscale Plasticity), applied to simulation results by dislocation dynamics. Focus is place on the stable/unstable of the dislocation systems in terms of FTMPbased incompatibility tensor field in particular via the attendant phase-space trajectories (PhS-Ts). The stable responses are shown to be manifested as the limit-like loci on the PhS-Ts, whereas the unstable responses mainly due to cross slip-driven vacancy-type debris loop generations are specifically represented by the incompatibility rate, i.e., the quantity well correlate the frequency of the debris loop formations. The incompatibility rate, used here as the ordinate of the PhS-Ts, can be interpreted as the creation/annihilation rate of edge dislocation pairs, in the FTMP-based context, which corroborates the correlation result.
\end{abstract}

\section{INTRODUCTION}

Crystalline materials under cyclic loadings show various variations of dislocation substructures in response to loading state [1, 2, 3, 4]. It is important to capture the evolution and morphology of the substructures properly because they dominate the mechanical responses of metals. The decisive method, however, has not been established due to its complex morphology. This is a major problem in the field of crystal plasticity.

Dislocation dynamics are commonly used for simulating meso-scale phenomena [5, 6, 7, $8,9]$. It can directly model dislocation sub-structures from experimental observations with less ad-hoc assumptions compared to continuum models. The configurations of dislocation ensembles are commonly captured by the dislocation density tensor $[10,11,12,13]$, however, using the tensor is not a sufficient way because it does not fully contain the information of configurations, i.e., it vanishes when counting dipoles or holds same value even whether two dislocations closely approach or not. Although the dislocation density tensor is useful for evaluating dislocation systems because its geometrical image is connected to the dislocation itself, the problem has been a long-standing issue in crystal plasticity.

To solve this problem, we have proposed the concept of FTMP (Field Theory of Multiscale Plasticity) [14, 15, 16, 17, 18, 19]. The FTMP is based on non-Riemannian plasticity [20, 21, 22], which represents defects in solids as the torsion tensor and the curvature tensor.

*Corresponding Author: ihara.shirou@mail.mm4.scitec.kobe-u.ac.jp 
The former is known to be translational defects, thus dislocations, while the latter expresses rotational defects; general defects such as vacancies, interstitials, and so on. The torsion tensor and the curvature tensor correspond to the dislocation density tensor and the incompatibility tensor, respectively. The key concept of the FTMP is to capture the evolution of dislocation ensembles as evolving inhomogeneity, i.e., the inhomogeneous field can be captured by incompatibility tensor since the tensor holds configurational aspects of dislocation ensemble. We have studied collapsing mixed dislocation walls [23], GNBs (Geometrically Necessary Boundaries) [24], and lath walls [25] by using the dislocation dynamics and evaluated based on the FTMP. These results show that the FTMP-based evaluation enables us to discuss the stability or instability of dislocation substructures.

The substructures, especially PSBs (Persistent Slip Bands) is a dominant factor of crack initiation [26, 27, 28, 29, 30, 31]. In the PSBs, which consist of dense edge dislocation dipole walls and dislocation poor channels, the dislocations move with back-and-forth motion under cyclic loading. They annihilate each other when a glide dislocation loop from one wall reaches another dislocation loop from an opposite wall, which generate vacancies or interstitials. These outcomes diffuse into matrix and cause intrusions or extrusions [26, 32]. Our earlier study shows that the vacancies diffuse to surfaces through the dislocation walls even if they generate from either the dislocation walls or the channels [33]. Thus, the PSBs are media of vacancy transfer, leading to crack initiation. The direct simulations and evaluations of the PSBs could be essential for multiscale modeling of fatigue simulations.

In this paper, we simulate the PSBs and apply the FTMP-based scheme to those. Our aim is to study dislocation interactions of PSBs and quantitatively evaluate these phenomena. This paper is organized as follows. In Section 2, we describe the mathematical foundations regarding to the FTMP-based evaluations. Sections 3 and 4 discuss PSB models in FCC and BCC, respectively, mainly focusing on the stability of the system and the effect of cross slip.

\section{THEORETICAL BACKGROUND}

Field theory of plasticity (FTMP) aims at explicitly describing evolutionary aspects of deformation-induced inhomogeneities in materials, putting its major basis on the nonRiemannian plasticity, i.e., differential geometrical field theory, advocated originally by Kazuo Kondo [22]. In FTMP, the attendant flow-evolutionary law, holding between the incompatibility tensor and the energy-momentum tensor fluctuation, has been demonstrated to control the field evolutions in many respects, although it is still a working hypothesis. The incompatibility tensor, among others, plays exclusively important roles, which allow reproductions of experimentally observed dislocation substructures simply by introducing the associated degrees of freedom into the constitutive model to be used via the hardening law in the crystal plasticity-implemented FEM (CP-FEM).

The incompatibility tensor is defined as,

$$
\eta_{i j}=\epsilon_{i k l} \epsilon_{j m n} \partial_{k} \partial_{m} \varepsilon_{l n}^{p}
$$

where $\boldsymbol{\epsilon}_{\boldsymbol{i} \boldsymbol{k} \boldsymbol{l}}$ is permutation tensor, $\varepsilon_{i j}^{p}$ is plastic strain tensor, $\partial_{k}=\partial / \partial x_{k}$, and Einstein convention is used. Equation (1) means that the incompatibility tensor reflects the deviation from the compatibility condition. The dislocation density tensor is represented by using the plastic distortion tensor $\beta_{i j}^{p}$, as 


$$
\alpha_{i j}=-\epsilon_{i k l} \partial_{k} \beta_{l j}^{p}
$$

Since the plastic strain tensor is the symmetrized part of the plastic distortion tensor, i.e., $\varepsilon_{i j}^{p}=$ $\frac{1}{2}\left(\beta_{i j}^{p}+\beta_{j i}^{p}\right)$. Substituting it into Equation (2), we get

$$
\eta_{i j}=-\epsilon_{i|k l|}\left(\partial_{|k|} \alpha_{j l}\right)
$$

Equation (3) indicates that the incompatibility tensor is calculated from the curl operation of the dislocation density tensor, thus, it is a non-local tensorial quantity. Since the non-locality, the incompatibility tensor could involve the information of the configuration of dislocation systems, which the dislocation density tensor does not hold.

Extended definition of the incompatibility tensor in four-dimensional (4D) space-time [19] is used in formulating the flow-evolutionary working hypothesis. Based on the divergence-free condition for the extended incompatibility tensor,

$$
\eta_{i j}=\epsilon_{i k l p} \epsilon_{j m n p} \partial_{k} \partial_{m} \varepsilon_{l n}^{p}
$$

we ultimately obtain,

$$
\eta_{\dot{K} K}=-\operatorname{div} \eta_{A 4} \text { with } \eta_{A 4}=-\left(\alpha_{B C}^{\cdot}\right)_{\text {skew }}
$$

where ()$_{\text {skew }}$ means skew-symmetrization operation, i.e., $\left(\alpha_{B C}\right)_{\text {skew }} \equiv \frac{1}{2}\left(\alpha_{B C}-\alpha_{C B}\right)$. This relationship can be readily derived from $\operatorname{div} \eta=\partial_{a}, \eta_{a b}=0$, i.e.,

$$
\frac{\partial \eta_{44}}{\partial x^{4}}+\frac{\partial \eta_{A 4}}{\partial x^{A}}=0 \Leftrightarrow \frac{\partial \eta_{K K}}{\partial t}=-\frac{\partial \eta_{A 4}}{\partial x^{A}}
$$

where the indices in lower-case letters represent those for 4D space-time i.e., $a, b=1,2$, 3, and 4 (time), while those in capital letters in Equation (4) express the "spatial" counterparts, e.g., A, B C=1,2,3. In the above, $\eta_{44}=\epsilon_{4 k l p} \epsilon_{4 m n p} \partial_{k} \partial_{m} \varepsilon_{l n}^{p}=\eta_{K K}$ is used. The physical meaning of Equation (4) becomes clear when we consider the global form of it, together with the divergent theorem, i.e.,

$$
\frac{\partial}{\partial t} \int_{v} \eta_{K K} d v=-\int_{v} \frac{\partial \eta_{A 4}}{\partial x^{A}} d v=-\int_{a} n_{A} \eta_{A 4} d a=\int_{a} n_{A}\left(\alpha_{B C}\right)_{s k e w}^{{ }_{s}} d a
$$

Based on which it reads the temporal change in $\eta_{K K}$ about (or balanced with) the dislocation flux across $d a$ ( $d x^{A}$ surface). Note that, more specifically, what the right-hand side it represents is the flux of edge dislocation rate $\left(\alpha_{B C}\right)_{\text {skew }}$, as understood from the characteristic of the dislocation density tensor whose off-diagonal component reflects edge dislocations. 
In this study, we obtain the dislocation density tensor from dislocations in a simulation cell, and calculate the incompatibility tensor by dividing the simulation cells into sub-cells and using least square method. Evolution of the PSBs is to be characterized by Phase-Space trajectories (PhS-Ts) of the incompatibility [17]. If a system demonstrates a closed-loop (limitcycle) on PhS-Ts, it is regarded as an oscillating system. The PSB models in this study are subjected to cyclic loading, therefore, their trajectories are closed-loci if they become stable because it states that the models just move in response to the external loading.

\section{PSB MODEL FOR FCC}

Stable responses are examined first in terms of the FTMP-based perspectives, by taking a FCC model as an example. The PSB ladder wall model system assumed is presented in Fig.1, where a pair of straight edge dislocation dipole walls are situated at the center of the unit cell, equipped with periodic boundary conditions in all directions, to mimic the PSB ladder structure. Cyclic shear stress is applied to the system with two levels of stress amplitude conditions of $\Delta \sigma=15,20,25[\mathrm{MPa}]$, according to

$$
\sigma_{13}=\Delta \sigma \sin \left\{\frac{(\text { Time Steps) } * \pi}{2500}\right\}
$$

up to five cycles. Here, "2500" represents the increment of simulation time steps for the half stress cycle, meaning 5,000-time steps per cycle. A use is made of the dislocation dynamics code, produced by Zbib et al. [34] in the present series of simulations. The material assumed is FCC copper, with material parameters; density $\rho=8940\left[\mathrm{~kg} / \mathrm{m}^{3}\right]$, shear modulus $\mu=46.1$ [GPa], Poisson's ratio $v=0.34$, and the magnitude of Burgers vector $b=2.56 \times 10^{-10}[\mathrm{~m}]$.

Before the cyclic stressing, the system is fully relaxed until the equilibrium configuration is reached. Figure 2 displays variation of dislocation density with simulation time steps during the relaxation process, together with the resultant configuration of the wall-constructing dislocations, where some fraction of them are already bowed out into the channel region. The bowed-put segments have the screw nature, whose to-and-fro motions are substantially responsible for carrying the plastic deformation of the system, i.e., PSB ladder structure [2].

Figure 3 shows series of snapshots during the first two cycles, comparing the three stress amplitudes. Commonly observed are the screw segments, coming out of the edge dipole walls toward inside the channel region at the center of the simulation cell during the first half cycle (1,000 steps), exhibit backward motions toward outside in the next half cycle (5,000 steps), while the remaining screw segments interact each other within the central channel, with some forming screw dipoles during their back-and-forth motions. Note that such screw dipoles basically act as obstacles against the other "flow-carrying" populations in general in the present context. Similar trend is confirmed in the following cycle (10,000 to 15,000 steps) but with some marked distinctions start to appear among the three; i.e., the case with $\Delta \sigma=15 \mathrm{MPa}$ yields almost commensurate configurational changes of the dislocations with the first cycle, whereas that with $\Delta \sigma=20 \mathrm{MPa}$ does not, where nearly synchronizing motions - the dislocations move back and forth without interactions in the channel - of them are observed at 15,000 steps, with the help of annihilations of redundant screw dipoles that are apt to interrupt the groupwise movements. The case with $\Delta \sigma=25 \mathrm{MPa}$ also demonstrates back and forth motion cyclically after 15,000 steps but continues to interact inside the channel region due to the highest external stress. 
$\left[\begin{array}{lll}1 & \overline{1} & \overline{2}\end{array}\right]$



Figure 1 Computational model of PSB for FCC. Only mobile dislocations are considered.

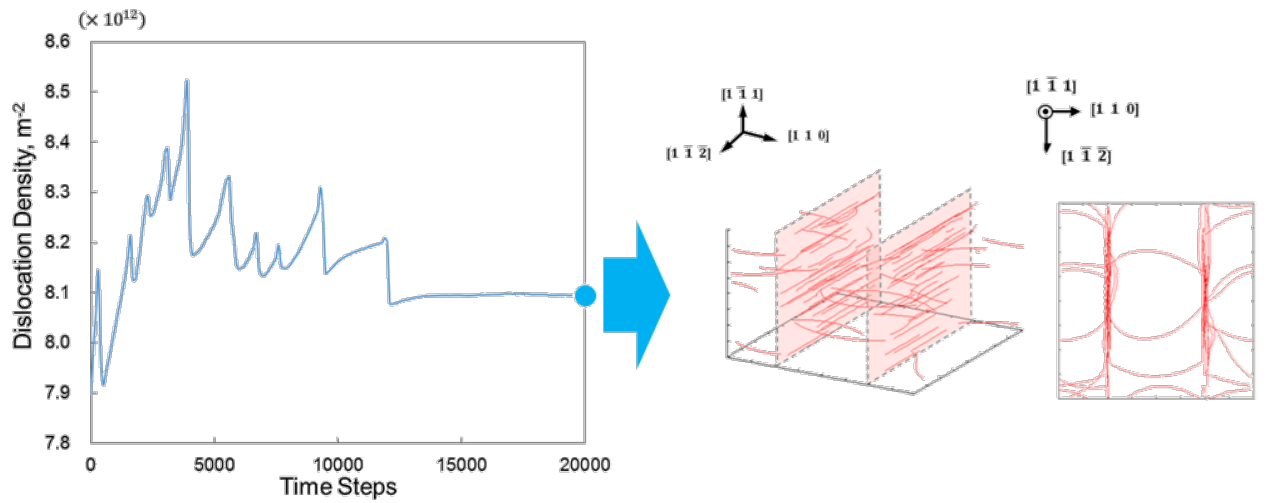

Figure 2 Simulated results for relaxation configuration of PSB model. Variation of dislocation density and configuration at which last step are shown.

Figure 4 plots the corresponding system-wise strain rate variation comparing the two conditions, each demonstrating transition toward a sort of equilibrated state. The case with $\Delta \sigma=15 \mathrm{MPa}$ yields rapid saturation during the first two cycles before reaching the equilibrium, while the other case with $\Delta \sigma=20 \mathrm{MPa}$ is shown to be still in the middle of transition even during the second cycle, after which exhibits rate change at higher strain rates due to the annihilation of the dipole, which is shown in Figure 3. Such relatively complex behavior simply stems from interaction with the dipoles. Increasing further the stress amplitude, on the other hand, the flow-carrying dislocations tend to move rather in unison, as confirmed in the case of $\Delta \sigma=25 \mathrm{MPa}$, because such high enough external stress can facilitate them to overcome the incidentally produced dipoles 


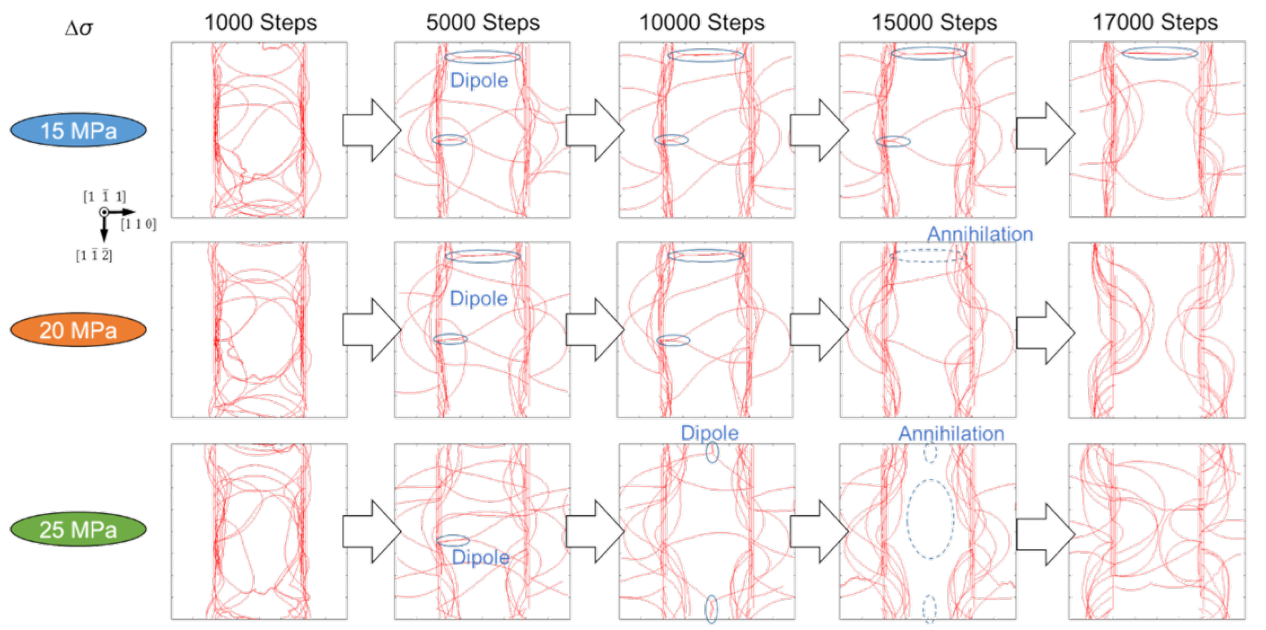

Figure 3 Snapshots of PSB models for both stress amplitude. Screw dipoles are generated, and they annihilate in lager stress amplitude model.

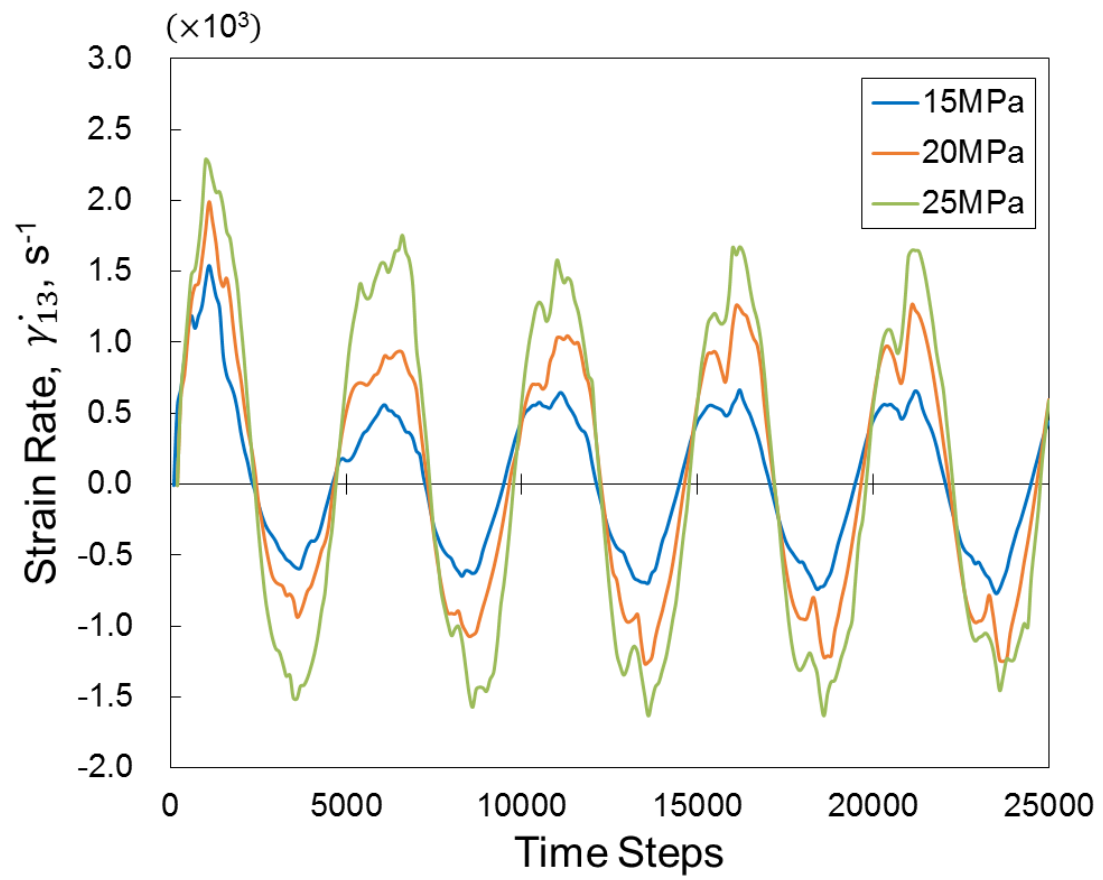

Figure 4 Variations of strain rate for FCC models. 
For the purpose of visualizing the above observations about the transitions toward their equilibrium states, we utilize here the FTMP-based phase-space trajectory (PhS-T) [17] of the incompatibility tensor field $\eta_{K K}$, defined as the plot between $\eta_{K K}$ and its change rate (time derivative) $\left(\eta_{K K}\right)$. Figure 5 displays thus obtained PhS-Ts for the three stress amplitude conditions, while the individual variations of the abscissa and the ordinate, i.e., $\eta_{K K}$ and $\left(\eta_{K K}\right)$. with time steps are compared in Figures 6(a) and (b), respectively. Readily noticed from Figure 5 is that all of them ultimately yield limit cycle-like loci, i.e., dynamically stable/steady states, but with mutually distinct manners. The PhS-T for $\Delta \sigma=15 \mathrm{MPa}$ rapidly reaches the limit-cycle-like state, after touring around the center of it, whereas that for $\Delta \sigma=20 \mathrm{MPa}$ takes more time, following a staggering track before reaching the steady state, which finally yields almost overlapping loci between the 4th and 5th cycles. Increasing the stress amplitude up to $\Delta \sigma=25 \mathrm{MPa}$, on the other hand, results again in the closed locus shortly, but not with mutual overlaps. The cycle-wise extracts for $\Delta \sigma=25 \mathrm{MPa}$ are displayed in the right row in the figure, from which we notice each contains large and small loops, while roughly maintaining the center. This rather complex behavior in the $\Delta \sigma=25 \mathrm{MPa}$ case mainly attributes to a pulsatinglike, quasi-periodic variation in the incompatibility rate $\left(\eta_{K K}\right)^{\text {; }}$, as confirmed in Figure 6(b), while the center of oscillation for $\eta_{K K}$ in Figure 6(b) has already reached a saturation during the first two cycles.

To be concluded tentatively based on the above PhS-T visualizations is that high enough external cyclic stress tends to promote complex but synchronized motions of the flow-carrying populations of dislocations, where the complexity is majorly attributed to the attendant formations and the following resolutions of the screw dipoles, as well as the interactions against them, frequently taken place in the channel regions. Such complexity is demonstrated further to be manifested as the pulsating-like variations in the incompatibility rate. Note, the incompatibility rate can be paraphrased as the conservation of the rate of edge dislocation pairs, as defined in Equation (4), which allows it to be interpreted also as variations of those brought about by the localized interactions with screw dipoles, i.e., trapping and unzipping against them, in the present context. 



Figure 5 Phase-space trajectories for PSB models, comparing three stress amplitude conditions.
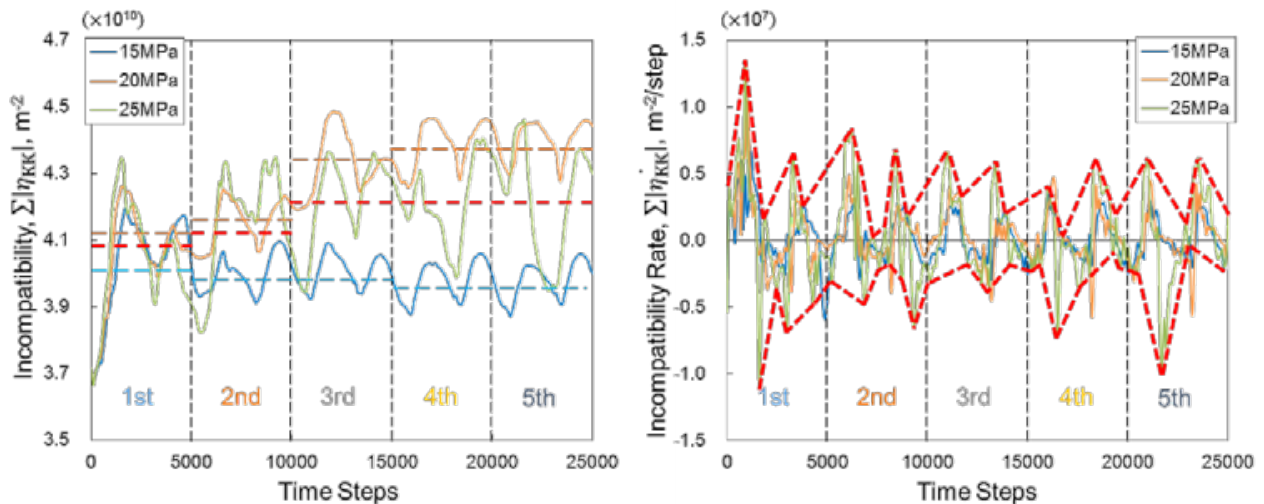

Figure 6 Variations of incompatibility and its rate for PSB models, comparing three stress amplitude conditions. 


\section{PSB MODEL FOR BCC}

As demonstrated above, stable PSBs tend to exhibit limit cycle-like closed loci on the PhS-T. Here, we examine the effect of the cross-slip frequency on the PhS-T in the case of a BCC system, assuming $\alpha$-Fe. To this end, three levels of cross slip frequencies are considered, i.e., high, low and without it, where high/low ratio is controlled by the critical stress with one order difference in the present series of simulations.

Figure 7 displays the simulation model used, where a single edge dipole wall is placed at the center of the simulation cell with periodic boundaries, consisting of a mobile segment in the middle of the wall otherwise immobile, in order to focus on the targeted effect. Stress cycle with amplitude of $\Delta \sigma=200[\mathrm{MPa}]$ is applied based on Equation (4) with 5000-step-period. Material parameters used in the current set of simulations are as follows: density $\rho=7630$ $[\mathrm{kg} / \mathrm{m} 3]$, shear modulus $\mu=53.8$ [GPa], Poisson's ratio $\nu=0.334$, and the magnitude of Burgers vector $b=2.485 \times 10^{\wedge}(-10)[\mathrm{m}]$.

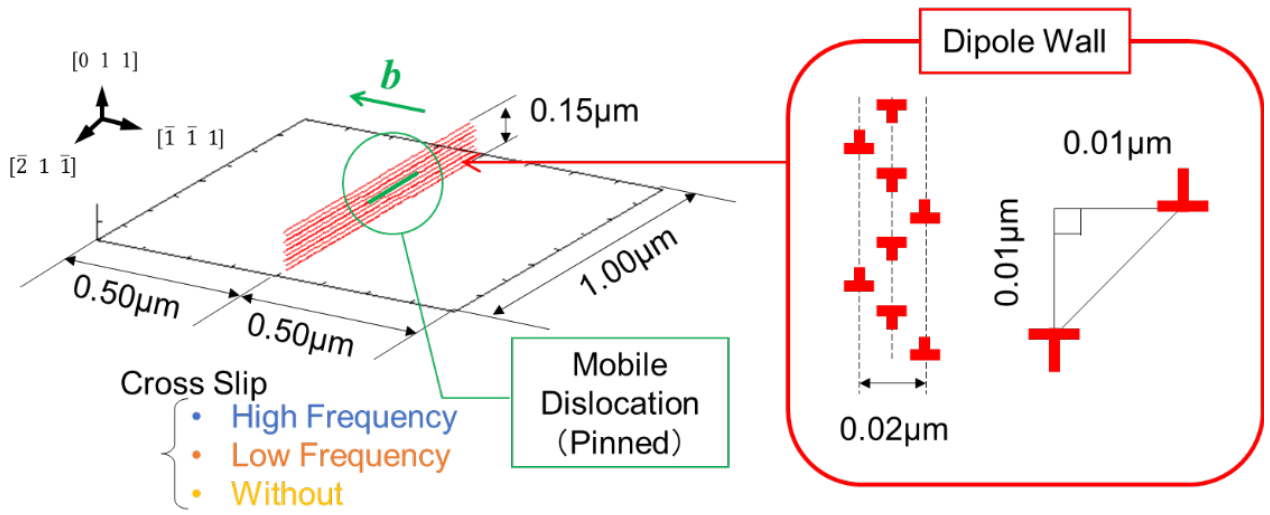

Figure 7 Computational model of PSB for BCC. For computational efficiency, one edge dipole wall is considered and only a pinned edge dislocation can glide.

Figure 8 compares series of snapshots ((a) through (f) (final)) among the three cases, together with their corresponding equivalent stress contours. By comparing with the case without cross slip (WO), we clearly confirm that the cross-slip effect is mainly manifested as complex interactions to take place in the channel regions in both sides of the wall, leaving ultimately many debris loops. The frequency of it increases with increasing cross slip frequency.

Closer look at the formation process of the debris loops in HF case reveals that the distinction starts to occur after the 1st cycle (b), meaning cross slip occurs somewhere during the first extension (a), which triggers further cross slips accompanied by local annihilations, eventually causing debris loops ((c) and after). This trend can be cross-checked in looking at the LF and WO cases, where the same but smaller number of debris loop formations also take place in conjunction with more stable to-and-fro motion of the bowing out segment for the former, while with no such event at all for the latter. These differences are quantitatively represented in Figure 9, where the variations of the number of debris loops are shown. Here, the debris loop is defined as a loop consisting of less than or equal to 10 nodes, deducing from the results. 



Figure 8 Snapshots of simulated results of PSB models for BCC. Cross slip causes generation of debris loops in channel, together with corresponding equivalent stress contours

Further details of the debris loop formation process observed for the HF model, for instance, are displayed in Figure 10 in a step-by-step manner via simulation snapshots. Here, the distinction among the slip planes that each dislocation segment belongs to is indicated by color variations.

The initially extended segment cross slips already at the late stage of the first forward loading (from red to green), as roughly observed in Figure 10, which brings about foldingback of the cross-slipped segment as soon as the following load reversal starts. During the early stage of it, two-step annihilation with the folded-up part takes place, eventually generating a large loop extending over four slip planes (red, green, orange and blue) left behind. Under the progressing reversed loading, the loop is transformed into a needle-like shape composing majorly of edge segments extending on the two slip planes (red and green) out of the four, via two more annihilation steps against the screw parts. 


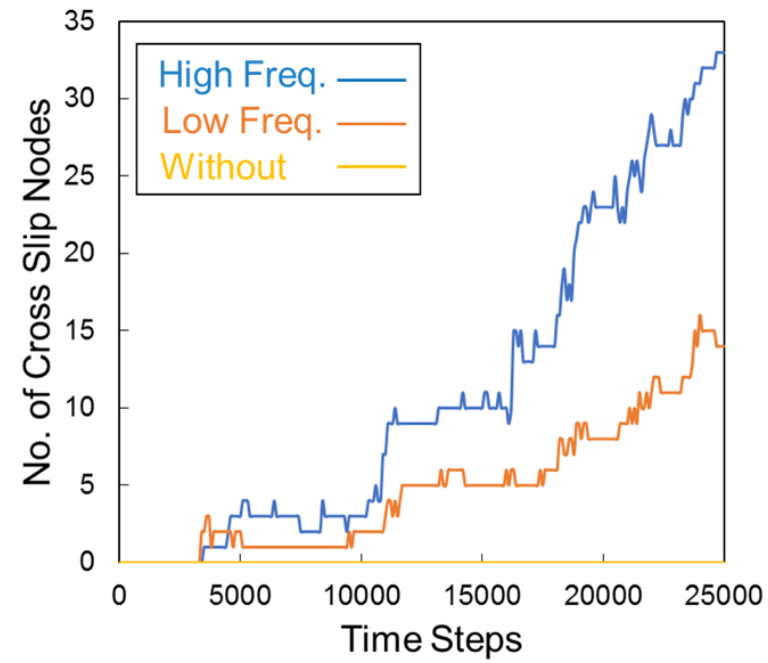

Figure 9 Variation of the number of debris loops.

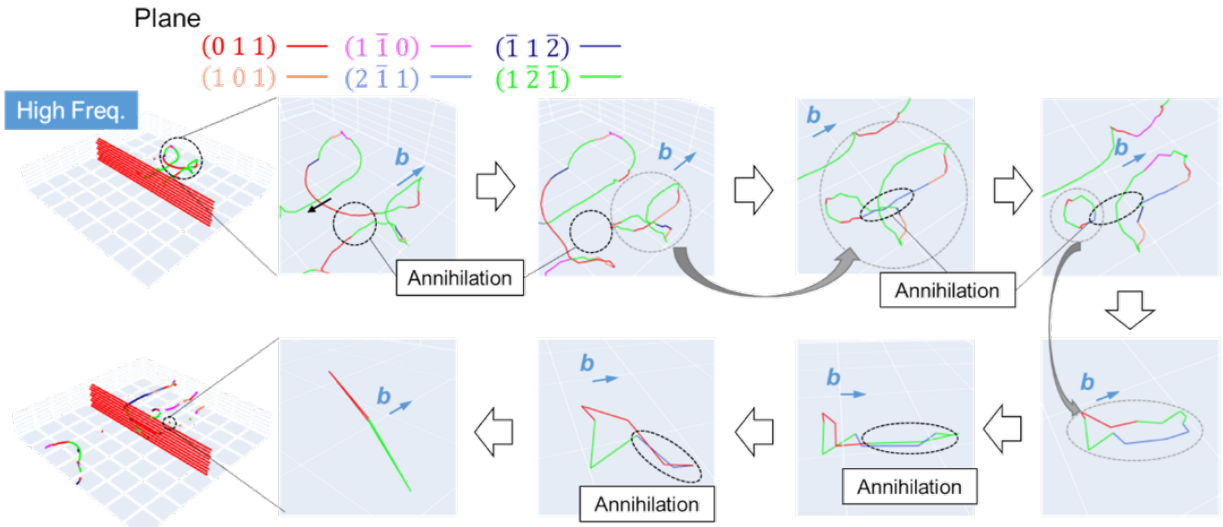

Figure 10 Snapshots of generation process of debris loops. When reversed loading, head of dislocations annihilates with other dislocations, leaving dislocation loops. Such generated dislocation loops repeat cross slip and annihilation with their dislocations. Finally, edge dislocation loops are left in channel.

Thus, formed edge loops potentially possess the nature of vacancies or interstitials depending on the Burgers vector-dislocation line relationship. Since the present case yields for the attendant edge pair the former, i.e., vacancies, the frequency of the cross slip is turned out to be a significant controlling factor of the vacancy generations associated with the PSB ladder structures. Figure 11 shows relationship between the number of cross slip nodes and that of the formed debris loops, clearly demonstrating a positive correlation between the two, regardless of the case we set in the present study. 




Figure 11 Relationship between the number of debris loops and the number of cross slip nodes.

Let us now see how we observe the above on the corresponding PhS-Ts, compared in Figure 12. The HF model exhibits rather stable loci, stagnated around a relatively large incompatibility value on the abscissa, accompanied by periodically increasing fluctuating rate of it. Contrary to this, the LF case yields unfixed/decreasing incompatibility, wandering toward the left on the abscess, while the incompatibility rate does not so fluctuate. The WO case, on the other hand, tends to converge eventually to a limit cycle like behavior that resembles those presented in Figure5 for FCC metal, as expected, around the commensurate value of the incompatibility for the HF case.

In the above, the incompatibility on the abscissa represents the amount of the shear deformation (strain amplitude or range) under the prescribed stress cycle in the present series of simulations, whereas the incompatibility rate on the ordinate can count how often and to what extent the dislocation segment motions involved are interrupted. Therefore, the apparently meta-stable PhS-Ts for the HF case indicate a sort of dynamically balanced annihilations and multiplications of dislocations ceaselessly taking place during the flowcarrying processes, accompanied by efficient productions of vacancy-type debris loops as the by product. In other words, a sufficient number of debris loop productions is implied to stabilize not only the flow resistance but also the flow-carrying ability of the PSB ladder structure. Insufficient debris-loop formations, on the other hand, as in the case of the LH model, tend to result both in unstable flow-carrying and lower flow resisting capabilities of the structure.

The argument described above implies the incompatibility rate as a candidate for characterizing the flow-carrying capability of the PSB ladder walls based on the discussions about the "dynamic equilibrium" of annihilation and multiplication processes in conjunction with the debris loop formations 


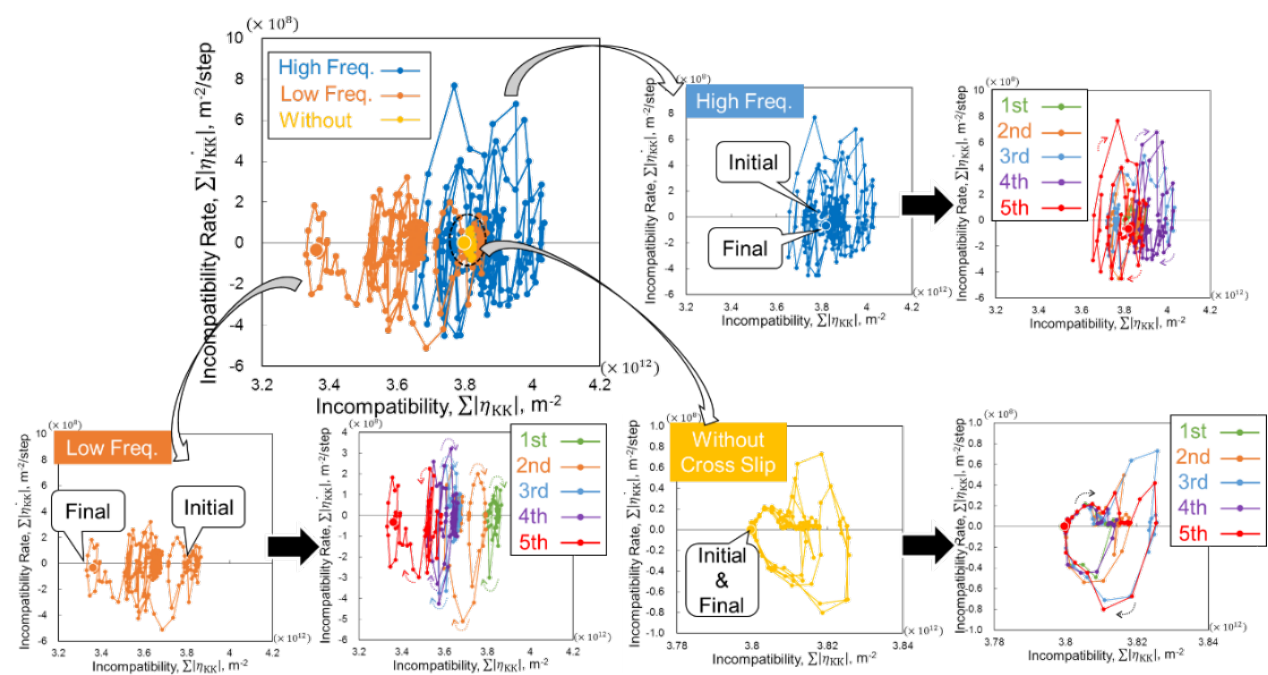

Figure 12 Phase-space trajectories of PSB models for BCC. The trajectories of each cycle are also shown for three models. Cross slip disturbs configuration of trajectories, which are originally showing closed loop.

As pointed out in conjunction with Equation 4, the incompatibility rate $\eta_{K K} \cdot$ dictates $\operatorname{div}\left(\alpha_{B C}\right)_{\text {skew }}^{*}$, which may safely be interpreted as the conservation of the rate of the edge dislocations, including edge dipoles, i.e., debris loops in the present context. Figure 13(a) compares variation of $\left(\eta_{K K}^{\cdot}\right)$ along with simulation time steps among the three conditions, where the HF case in particular exhibits exclusively peaky as well as growing trends among others, that corroborates this interpretation.

Figure 13(b) correlates the number of debris loops with the amplitude of the incompatibility rate for the corresponding trajectory cycle, where the amplitude is calculated for each half cycle, as shown in Figure 13(b). The figure demonstrates a positive correlation between the two quantities regardless the case. This result can be rephrased further as that the incompatibility rate amplitude measures the vacancy-type debris loop formation capability within PSB ladder structures. Anticipated vacancy distributions based on the corresponding debris loops for the HF and LF cases are shown in Figure 14, demonstrating that vacancy formations are apt to occur not only within the ladder wall but also in the channel regions.

Vacancies produced associated with the PSB ladder walls are supposed further to play cooperative roles in promoting fatigue crack initiation processes [28, 32]. A separate series of diffusion simulations reveal that vacancy flux produced within the channel tends to flow into the wall region first and subsequently diffuse toward the surface along both edges of the PSB, promoting resultantly the "intrusion" evolutions thereof [33]. Therefore, the relationship

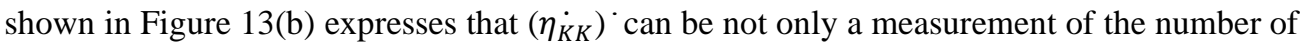
vacancies, but also a practical parameter for modeling the fatigue phenomena. 
(a)

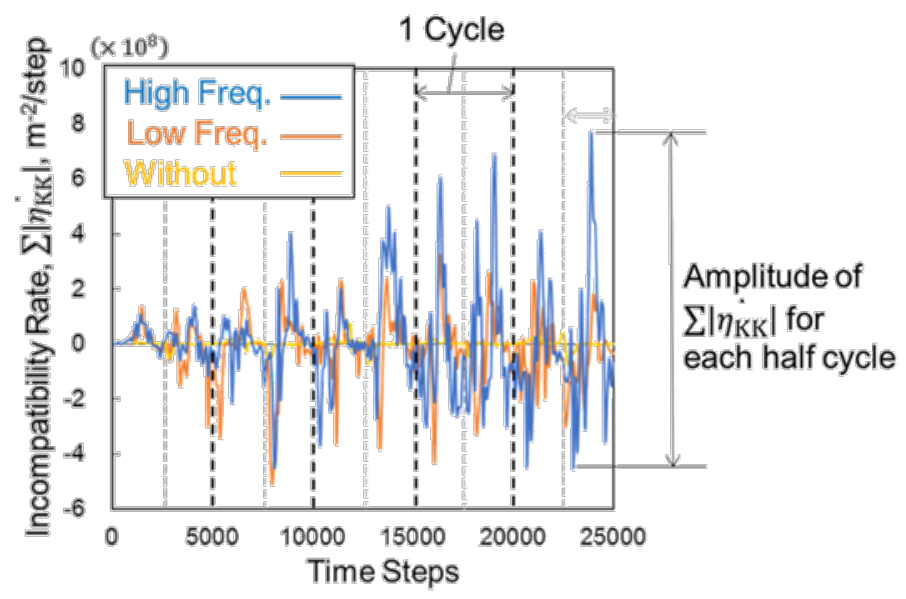

(b)



Figure 13 (a) Variation of $\sum\left|\dot{\eta_{\mathrm{KK}}}\right|$ and (b) correlation of the number of generated debris loops and amplitude of $\sum\left|\eta_{\mathrm{KK}}^{\cdot}\right|$. 




Figure 14 Anticipated vacancy distributions based on those of debris loops, comparing between high and low frequency cross slip conditions.

\section{CONCLUSIONS}

This study extensively discussed discrete dislocation motions associated with ladder-wall structures that consist PSBs (persistent slip bands), known to be ultimately responsible for fatigue crack initiation in general, in the light of FTMP (field theory of multiscale plasticity), i.e., via incompatibility evolutions. Use was made for the evaluations of the phase-space trajectory (PhS-T) scheme with respect to the incompatibility tensor field, i.e., the plot of incompatibility tensor versus incompatibility rate. The incompatibility rate, based on FTMP, represents conservation of the rate of the edge dislocation loops. We also investigated the effect of the cross slip by changing the frequency of it. Major results obtained are summarized as follows.

(1) The stable PSBs are shown to be characterized by limit cycle-like loci, with external stress-dependent transient states before reaching them. The transitions are mainly controlled by screw dipole formations and the attendant interactions against them. Large enough stress amplitude tends to allow the flow-carrying dislocation motions to be in a synchronized one, resulting in steady PhS-Ts even though dislocation interactions frequently occur. 
(2) The frequency of the cross slip greatly affects stability/instability of the ladder-wall structure in the sense that it promotes debris loop formations that further interrupting smooth flow-carrying motions of dislocations. Since the debris loops basically organized into edge components belong to separate slip planes, formations of them are interpreted as equivalent to those of vacancies.

(3) The unstable responses are demonstrated to exhibit also limit cycle-like loci eventually but with temporarily fluctuating incompatibility rate, as visualized based on the PhS-T presentations. Consequently, positive correlations are found between the amplitude of the incompatibility rate and the number of generated debris loops.

(4) It is additionally shown that the incompatibility rate-loop formation relationship in (3) can allow us to make use of the present finding in modeling continuum mechanics-based descriptions of PSB ladder structures aiming at simulating fatigue crack initiation processes, e.g., based on FTMP-implemented CP (crystal plasticity) -FEM.

\section{REFERENCES}

[1] D. Kuhlmann-Wilsdorf and N. Hansen, "Geometriclly Necessary, Incidental and Subgrain Boundaries," Scripta Metall. Mater., vol. 25, no. 7, pp. 1557-1562, 1991.

[2] S. Suresh, Fatigue of Materials, Cambridge University Press, 1998.

[3] C. Hong, X. Huang and G. Winther, "Dislocation Content of Geometrically Necessary Boundaries Aligned with Slip Planes in Rolled Aluminium," Phil. Mag. A, vol. 93, no. 23, pp. 3118-3142, 2013.

[4] E. Tanaka, S. Murakami and M. Ooka, "Dependence of strain-Hardening under Multiaxial Non-Proportional Cyclic Plastic Deformation on Geometry History of Plastic Strain Trajectory," Trans. JSME A, vol. 51, no. 468, pp. 1941-1950, 1985.

[5] Arsenlis, W. Cai, M. Tang, M. Rhee, T. Oppelstrup, G. Hommes, T. G. Pierce and V. V. Bulatov, "Enabling Strain Hardening Simulations with Dislocation Dynamics," Modelling Simul. Mater. Sci. Eng., vol. 15, no. 6, pp. 553-595, 2007.

[6] V. V. Bulatov and W. Cai, Computer Simulations of Dislocations, Oxford University Press, 2006.

[7] N. M. Ghoniem, S. H. Tong and L. Z. Sun, "Parametric Dislocation Dynamics: A Thermodynamics-based Approach to Investigations of Mesoscopic Plastic Deformation," Phys. rev. B, vol. 61, no. 2, pp. 913-927, 2000.

[8] L. Kubin, G. Canova, B. Devincre, V. Pontikis and Y. Brechet, "Dislocation Microstructure and Plastic Flow: 3D Simulation," Solid State Phenomena, vol. 23\&24, pp. 455-472, 1992.

[9] H. M. Zbib, M. Rhee and J. P. Hirth, "3D Simulation of Curved Dislocations: Discretization and Long-range Interactions," in Advances in Engineering Plasticity and its Applications, T. Abe and T. Tsuruta, Eds., Pergamon, 1996, pp. 15-20.

[10]K. Aifantis, J. Senger, D. Weygand and M. Zaiser, "Discrete Dislocation Dynamics Simulation and Continuum Modeling of Plastic Boundary Layers in Tricrystal Micropillars," Mater. Sci. Eng., vol. 3, p. 012025, 2009. 
[11]Y. Zhu and Y. Xiang, "A Continuum Model for Dislocation Dynamics in Three Dimensions using the Dislocation Density Potential Functions and its Application to Micro-Pillars," J. Mech. Phys. Solids, vol. 84, pp. 230-253, 2015.

[12] R. E. Jones, J. A. Zimmerman, G. Po and K. K. Mandadapu, "Comparison of Dislocation Density Tensor Fields Derive from Discrete Dislocation Dynamics and Crystal Plasticity Simulations of Torsion," J. Mater. Sci. Res., 2016.

[13] J. F. Nye, "Some Geometrical Relations in Dislocated Crystals," Acta Metall., vol. 1, pp. 153-162, 1953.

[14] Y. Aoyagi and T. Hasebe, "New Physical Interpretation of Incompatibility Tensor and Its Application to Dislocation Substructure Evolution," Key Materials Engineering, vol. 340, no. 341, pp. 217-222, 2007.

[15] T. Hasebe, "Continuum Description of Inhomogeniously Deforming Polycrustalline Aggeregate ased on Field Theory," IUTAM Symposium on Mesoscopic Dynamics of Fracture Pricess and Materials Strength. Edited by kitagawa, H. and Shibutani, Y., Kluwer Academic Publishers, pp. 381-390, 2004.

[16] T. Hasebe, "Interaction Fields Based on Incompatibility Tensor in Field Theory of Plasticity -Part I: Theory-," IMMIJ, vol. 2, no. 1, pp. 1-14, 2009.

[17] T. Hasebe, "Interaction Fields Based on Incompatibility Tensor in Field Theory of Plasticity -Part II: Application-," IMMIJ, vol. 2, no. 1, pp. 15-30, 2009.

[18] T. Hasebe, "Multiscale Crystal Plasticity Modeling based on Field Theory," CMES, vol. 11, no. 3, pp. 145-155, 2006. doi:10.3970/cmes.2006.011.145

[19] T. Hasebe, M. Sugiyama, H. Adachi, S. Fukutani and M. Iida, "Modeling and Simulations of Experimentally-Observed Dislocation Substructures Based on Field Theory of Multiscale Plasticity (FTMP) Combined with TEM and EBSD-Wilkinson Method for FCC and BCC poly/Single Crystals," Mater. Trans., vol. 55, no. 5, pp. 779-787, 2014.

[20] K. Kondo, "Derivation of the Differential Equations of Yielding from General Variational Criteria in Analogy with General Relativity Theory and an Extension Thereof to Problems involving Critical Temperature," RAAG Memoirs of Unifying Study of Basic Problems in Engineering and Physical Science by Means of Geometry, Vols. 3(D-XII), pp. 215227, 1962.

[21]K. Kondo, "Energy at Plastic Deformation and the Criterion for Yielding," RAAG Memoirs of Unifying Study of Basic Problems in Engineering and Physical Science by Means of Geometry, vol. 1, pp. 484-494, 1955.

[22] K. Kondo, "Non-Riemannian Geometry of Imperfect Crystals from a Macroscopic Viewpoint," RAAG Memoirs of Unifying Study of Basic Problems in Engineering and Physical Science by Means of Geometry, vol. 1, pp. 458-469, 1955.

[23] T. Hasebe and T. Naito, "FTMP-based 4D Evaluations of Discrete Dislocation Systems," in New Frontiers of Nanometals (Proc. 35th Ris $\varnothing$ int. Symp. on Maters. Sci.), S. Faester, Ed., 2014, pp. 305-312. 
[24] S. Ihara and T. Hasebe, "FTMP-based Simulations and Evaluations of GeometricallyNecessary Boundaries (GNBs) of Dislocation," Int. Jnl. of Multiphysics, vol. 13, no. 3, pp. 253-268, 2019.

[25] S. Ihara and T. Hasebe, "FTMP-based Evaluations of Dislocation Wall Structures," Proc. of 10th Japan-China Bilateral Symp. on High Temperature Strength of Materials, pp. 304310, 2019.

[26] U. Essmann, U. Gösele and H. Mughrabi, "A Model of Extrusions and Intrusions in Fatigued Metals I. Point-Defect Production and the Growth of Extrusions," Phil. Mag. A, vol. 44, no. 2, pp. 405-426, 1981.

[27] K. Differt, U. Esmann and H. Mughrabi, "A Model of Extrusions and Intrusions in Fatigued Metals II. Surface Roughening by Random Irreversible Slip," Phil. Mag. A, vol. 54, no. 2, pp. 237-258, 1986.

[28] J. Polák, "On the Role of Point Defects in Fatigue Crack Initiation," Mater. Sci. Eng., vol. 92, pp. 71-80, 1987.

[29] J. Polák, M. Petrenec and J. Man, "Dislocation Structure and Surface Relief in Fatigued Metals," Mater. Sci. Eng. A, vol. 400, no. 401, pp. 405-408, 2005.

[30] J. Polák and J. Man, "Fatigue crack initiation - The role of point defects," Int. J. Fatigue, vol. 65, pp. 18-27, 2014.

[31]E. A. Repetto and M. Ortiz, "A Micromechanical Model of Cyclic Deformation and Fatigue-Crack Nucleation in f.c.c. Single Crystals," Acta Mater., vol. 45, no. 6, pp. 25772595, 1997.

[32] J. Polák, J. Man and K. Obrtlík, "AFM evidence of surface relief formation and models of fatigue crack nucleation," Int. J. Fatigue, vol. 25, no. 9-11, pp. 1027-1036, 2003.

[33] S. Nakatani, "FTMP-based Modeling and Simulation for Fatigue Crack Initiation Process from Persistent Slip Band," Master Thesis, Kobe Univ., Japan, 2012.

[34] H. Zbib, T. De La Rubia, M. Rhee and J. Hirth, "3D Dislocation Dynamics: Stress-Strain Behavior and Hardening Mechanisms in FCC and BCC Metals," Journal of Nuclear Materials, vol. 276, no. 1-3, pp. 154-165, 2000.

[35] J. M. T. Thompson and H. B. Stewart, Nonlinear Dynamics and Chaos, John Wiley \& Sons, 1986. 